\section{Pathogenetic and clinical study of a patient with thrombocytopenia due to the p.E527K gain-of-function variant of SRC}

The SRC gene was the first proto-oncogene to be discovered. Its product SRC is a nonreceptor protein tyrosine kinase that is the prototype, and a ubiquitously expressed member, of the SRC family kinases. SRC has been investigated for decades in mouse and in vitro models: these studies have indicated that SRC signalling has a central role in many cellular functions and in oncogenesis. In platelets, SRC mediates signal activation pathways downstream different integrins and $G$ protein-coupled receptors. ${ }^{2}$ However, much remains to be understood about SRC functions in human megakaryocytes and platelets.

Recently, the first germline mutation in $S R C$ causing human disease was reported in two families. ${ }^{3,4}$ The heterozygous c. $1579 \mathrm{G}>\mathrm{A}$ variant, causing the p.E527K substitution in SRC, was characterized as a gain-of-function mutation resulting in a constitutively active SRC due to the enhanced autophosphorylation of its Tyrosine- 419 . This mutation was associated with a complex syndromic phenotype characterized by thrombocytopenia variably associated with facial dysmorphism, severe osteoporosis, autism, intellectual disability, premature edentulism, and adult-onset myelofibrosis with splenomegaly. ${ }^{3,4}$ It has been suggested that thrombocytopenia derives from defective megakaryocyte maturation. ${ }^{3}$ Here we report the investigation of a new unrelated individual carrying the p.E527K variant that provides additional information on the clinical and pathogenetic features of the disorder and the role of SRC in human megakaryocytes. The study was approved by the Ethic Committee of Pavia and conducted according to the Declaration of Helsinki. The patient's parents provided written informed consent.

Clinical and laboratory characterization. The proband was a 2-year-old male, born to healthy parents, referred for the investigation of congenital thrombocytopenia and excessive bleeding. Platelet count ranged from 55$100 \times 10^{9} / \mathrm{L}$ with no other cytopenias (Online Supplementary Table S1). He presented at age 3 days with bilateral cephalohematoma with jaundice, and since then has had a lifelong history of easy bruising, petechiae, and occasional moderate epistaxis. Moreover, at the age of 3 months he was diagnosed with spontaneous left periventricular hemorrhage resulting in mild right hemiparesis. Physical examination showed no pathological findings, except for the hemiparesis. Intellectual development and learning were normal. Examination of peripheral blood smears revealed platelet anisocytosis and macrocytosis and a reduced $\alpha$-granules content with about $30 \%$ of hypo- or agranular platelets (Online Supplementary Figure $S 1 A-C)$. The $\alpha$-granule defect was confirmed at ultrastructural analysis (Online Supplementary Figure S1D). Consistent with the $\alpha$-granule deficiency, the platelet amount of thrombospondin-1 and von Willebrand factor was reduced (Online Supplementary Figure S1E-F), and the surface exposure of P-selectin was defective after stimulation with all tested agonists (Online Supplementary Figure $S 1 G$ ). Study of platelet aggregation showed a defective response to collagen, whereas response to ADP and TRAP was normal (Online Supplementary Table S2). Platelet functional response to these agonists was also investigated as the induction of surface expression of the activated form of glycoprotein IIbIIIa: we found a signif- icantly reduced response to collagen and a very mild defect after stimulation with $\mathrm{ADP}$ and TRAP (Online Supplementary Figure $S 1 H$ ). Flow cytometry did not show any defects in the expression of the major platelet surface glycoproteins (Online Supplementary Table S3).

In order to investigate the genetic cause of the thrombocytopenia, we performed whole exome sequencing of the proband. The analysis identified the c. $1579 \mathrm{G}>\mathrm{A}$ variant in the $S R C$ gene (p.E527K substitution in SRC) in heterozygosis. ${ }^{3}$ No other potentially pathogenetic variants were found in the other genes known to be responsible for inherited thrombocytopenia. Sanger sequencing showed that the proband's parents, who had normal blood cell counts, did not carry the variant, which was therefore considered as de novo.

The clinical picture remained stable until the age of the 3.5 years, when the proband began to present unexplained, moderate hyporegenerative anemia (Online Supplementary Table S1). A bone marrow (BM) biopsy was therefore performed. Examination concluded for a hypercellular BM with moderate trilineage dysplasia, no excess blasts, and significantly increased reticulin (fibrosis grade 2 according to Thiele). ${ }^{5}$ Megakaryocytes (Mk) were numerous, irregularly distributed in intertrabecular areas, and represented at all stages of maturation with dysmegakaryopoiesis and some micromegakaryocytes. No signs of Mk emperipolesis were present.

Subsequently, anemia progressively worsened and required periodic red blood cell transfusions; we observed also a progressive decrease in platelet count and a mild splenomegaly (Online Supplementary Table S1). The search for a donor for hematopoietic stem cell transplantation was therefore started.

Pathogenetic studies. We first checked that the SRC p.E527K variant actually results in constitutive increased activation of SRC. Consistent with previous findings, ${ }^{3}$ SRC was in a hyperactivated state in the patient's resting platelets (Online Supplementary Figure S2). We also found increased activation of the tyrosine kinase FAK, a substrate of SRC involved in several mechanisms, such as cell adhesion and cytoskeleton reorganization (Online Supplementary Figure S2).

The experiments reported below were carried out on two different occasions, when the patient was 2.5 and 3.0 years old, and the results were compared with those obtained in three healthy controls. Given the suggested role of SRC in cell adhesion, ${ }^{7}$ we tested the ability of the patient platelets to interact with different proteins of the extracellular matrix (ECM). Mutant platelets showed significantly increased adhesion and spreading on fibrinogen, type I collagen, and von Willebrand Factor (Online Supplementary Figure S3), suggesting that SRC hyperactivation induces a generalized enhancement of platelet adhesion to the ECM.

We then differentiated in vitro $\mathrm{Mk}$ from peripheral blood progenitors of the patient according to a standard protocol ${ }^{8,9}$ At the end of the culture, the maturation profile of the patient's Mk was similar to that of controls (Figure 1A-C). Differently from previous findings, ${ }^{3}$ proplatelet formation (PPF) of mutant $\mathrm{Mk}$ in suspension liquid cultures was comparable to controls (Figure 1D-E). However, when Mk were let adhere to fibrinogen or type I collagen, two components of the BM ECM that regulate platelet formation, mutant $\mathrm{Mk}$ exhibited a markedly increased adhesion and spreading, often with aberrant morphology (Figure 2; Online Supplementary Figure S4). This prominent adhesion phenotype was associated with 
an increased number and density of podosomes, i.e., the actin-based focal adhesion structures that mediate $\mathrm{Mk}$ contact with ECM proteins (Figure 3A-C). ${ }^{3,10,11}$ Figure 3A shows that the active form of SRC, recognized by an antibody specific to phospho-Tyrosine-419, was closely associated with these adhesion structures, supporting the role of the constitutively active SRC in their formation. Importantly, in adhesion to fibrinogen, an ECM substrate that promotes $\mathrm{PPF}^{8,9}$ the increased spreading of the patient's Mk was associated with a significantly reduced extension of typical proplatelets (Figure 2A-B). PPF of mutant $\mathrm{Mk}$ adhering to ECM proteins had not been investigated in previous studies. ${ }^{3,4}$

Finally, Mk migration on ECM proteins toward a gradient of stromal cell-derived factor 1 (SDF1) is an essential mechanism for platelet production, as it allows $\mathrm{Mk}$ to reach the $\mathrm{BM}$ vascular structures in order to release proplatelets into the circulation. ${ }^{12}$ Using a modified transwell assay, we found that patient $\mathrm{Mk}$ presented a significantly impaired SDF1-driven migration both in adhesion to fibrinogen and type I collagen (Figure 3D-E).

The study of this patient contributes to elucidate the pathogenesis of SRC-related thrombocytopenia and the role of SRC in human Mk. We provide evidence that
A

B

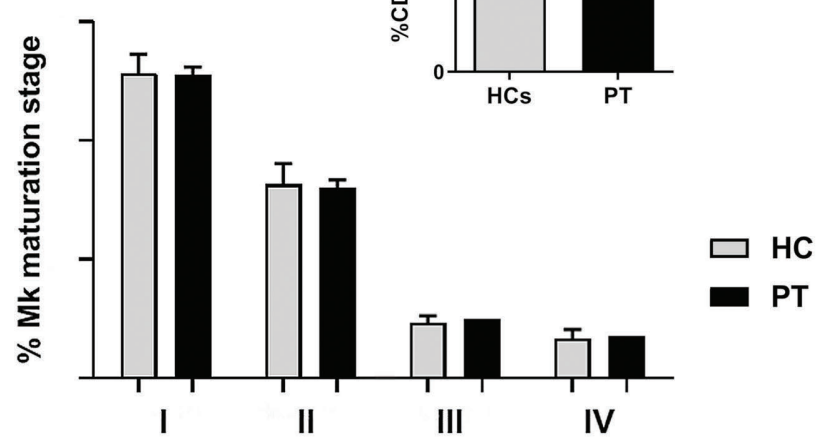

C
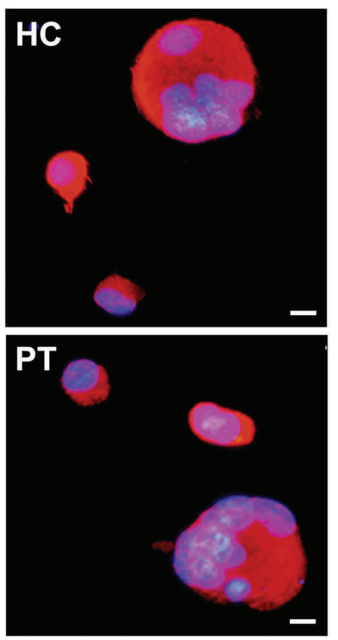

D

Healthy control

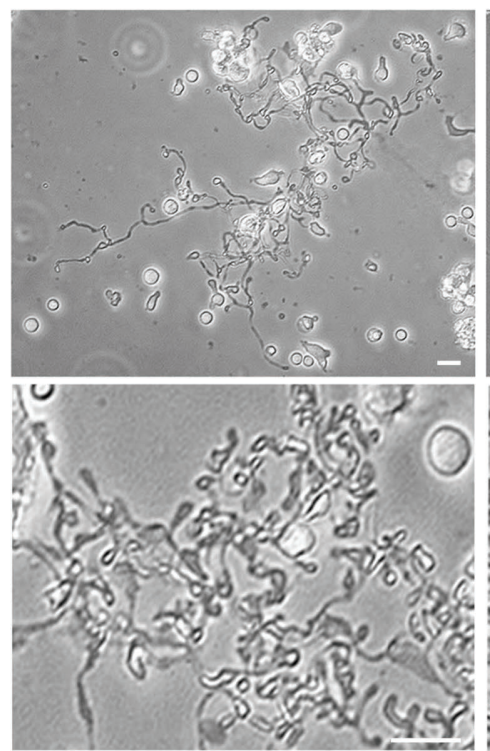

Patient

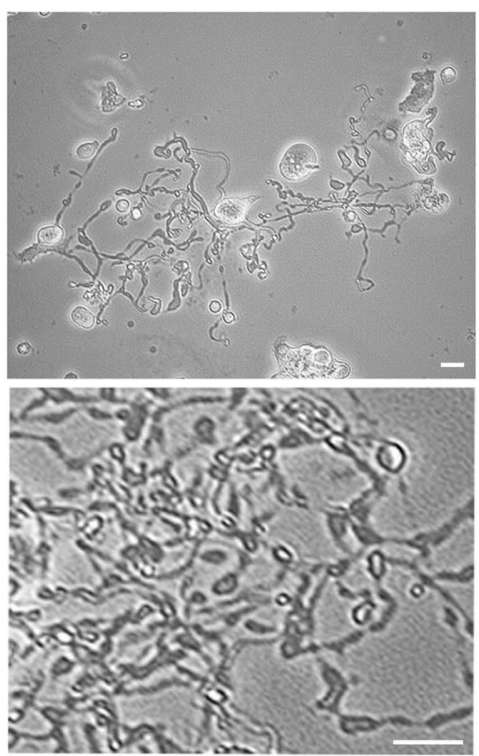

$\mathrm{E}$

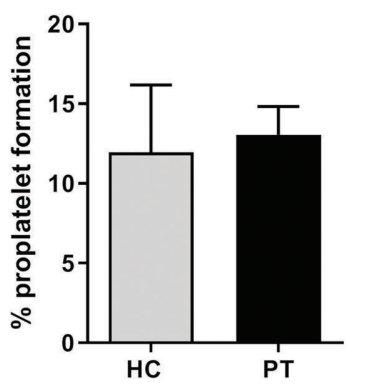

Figure 1. Analysis of maturation and of proplatelet formation in suspension liquid cultures of megakaryocytes of the investigated patient. Megakaryocytes (Mk) were differentiated from peripheral blood progenitors through 14-day culture. Samples of the patient (PT) were processed in parallel with those of three healthy controls $(\mathrm{HC})$. (A-C) Analysis of Mk maturation. (A) At the end of the culture, the proportion of mature Mk was measured by flow cytometry, as the percentage of CD41-positive cells co-expressing the CD42b antigen. ${ }^{9}$ (B,C) Mk were also cytospun onto slides and stained with an anti- $\beta 1$-tubulin antibody (red). Hoechst (blue) was used for counterstaining nuclei (C). Mk were then classified into maturation stages I to IV by morphological analysis according to standard criteria $^{16}$ (B). Overall, the maturation profile of the patient's Mk was not different from that of healthy controls. Scale bars: $10 \mu \mathrm{m}$. (D-E) Analysis of proplatelet formation in suspension liquid cultures, which measures the intrinsic ability of Mk to form proplatelets i.e., free from the engagement with proteins of the extracellular matrix. (D) Representative examples of proplatelet formation of Mk of the patient and controls (phase-contrast microscopy). Scale bars: $10 \mu \mathrm{m}$. (E) Proplatelet formation was quantified as the percentage of cells displaying at least one proplatelet with respect to the total number of cells. Overall, the rate of proplatelet formation and the morphology of proplatelets in suspension were similar in patient and controls. The data are expressed as means \pm standard deviation. 
thrombocytopenia derives from an altered interaction of Mk with the ECM components. In fact, mutant Mk presented normal PPF when cultured free from the engagement with ECM proteins; however, when plated on fibrinogen, they showed an abnormally increased adhesion that associated with impaired PPF and SDF1-driven migration. We conclude that a physiologically modulated SRC signalling in Mk is crucial for adhesion and migration on ECM components. In fact, pharmacological inhibition of SRC in mouse $M k$ induced decreased adhesion/spreading and defective migration; ${ }^{7}$ moreover, reduced activation of SRC in Mk of patients with thrombocytopenia due to PTPRJ loss-of-function variants also associated with defective migration. ${ }^{13}$ On the other hand, here we showed that SRC hyperactivation in human Mk causes increased adhesion/spreading, which also results in impaired SDF1-driven migration. The latter is probably due to altered turnover of focal adhesion structures, which is essential for migration. ${ }^{11}$ Since actin cytoskeleton reorganization after $\mathrm{Mk}$ interaction with the ECM is crucial for proplatelet extension, ${ }^{14}$ an altered cytoskeletal rearrangement upon $\mathrm{Mk}$ adhesion to fibrinogen, due to SRC constitutive activation, likely underlies the impaired PPF.
Concerning the clinical aspects, this case demonstrates that the disorder deriving from $S R C$ mutation can present as isolated, non-syndromic thrombocytopenia. Of note, the only feature common to all the reported patients is platelet anisocytosis and macrocytosis with a proportion of hypo- or agranular platelets (Online Supplementary Table S4). ${ }^{3,4}$ The other inherited thrombocytopenias that can present with this picture are thrombocytopenia due to $G F I 1 B$ mutations and gray platelet syndrome due to NBEAL2 variants, ${ }^{15}$ although in the latter the $\alpha$-granule defect is usually more pronounced. Therefore, these disorders should be considered in the differential diagnosis. A defective aggregation or activation response to collagen, albeit of varying degrees, was observed in most reported patients: this finding too could be considered a feature of the disorder. Similar to other cases, ${ }^{3}$ our patient presented a bleeding tendency more severe than expected based on the platelet count: the $\alpha$-granule deficiency and the impaired response to collagen could explain the excessive bleeding. Finally, the moderate thrombocytopenia observed at diagnosis evolved during childhood toward a picture characterized by transfusion-dependent anemia, severe thrombocytopenia, and trilineage BM dysplasia with significant fibrosis. The disorder can
A
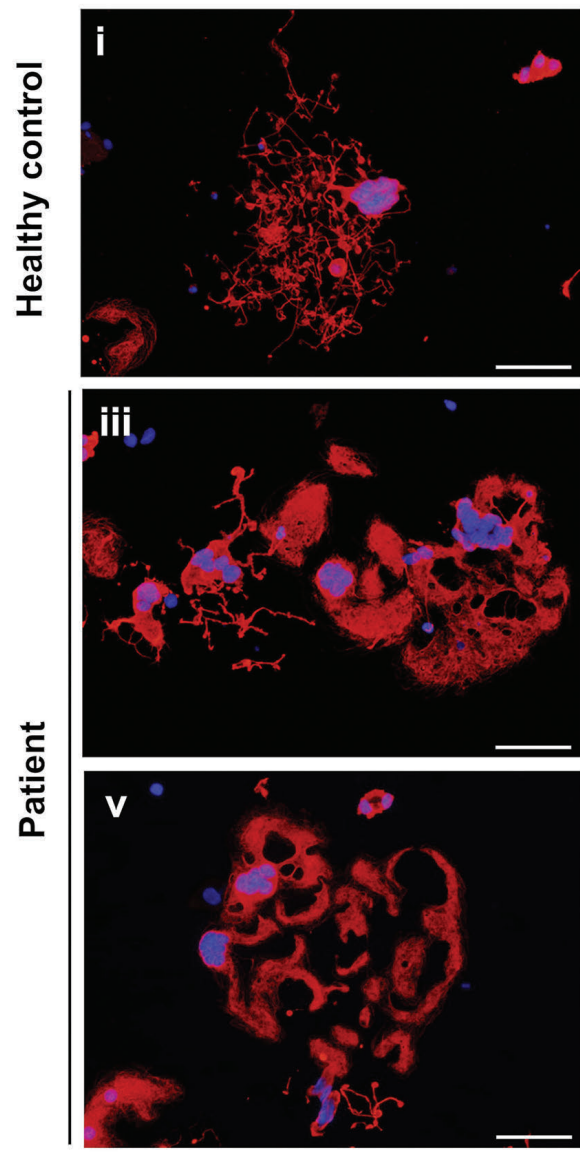
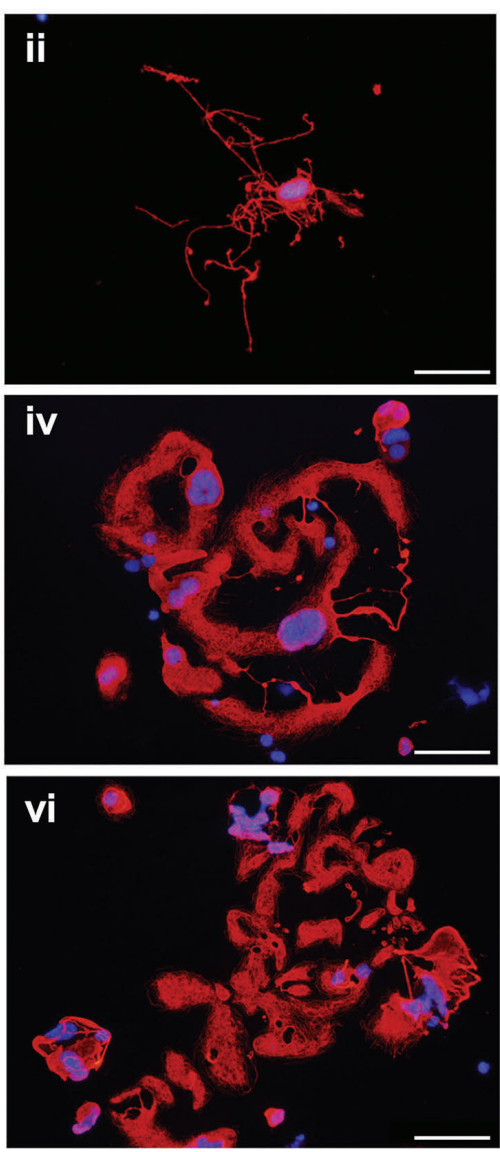

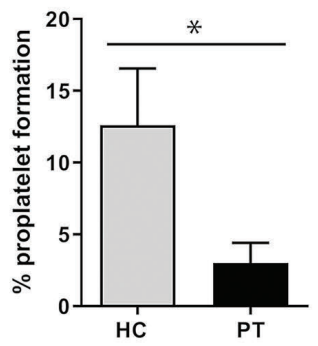

C

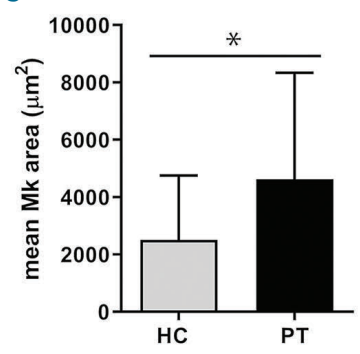

D

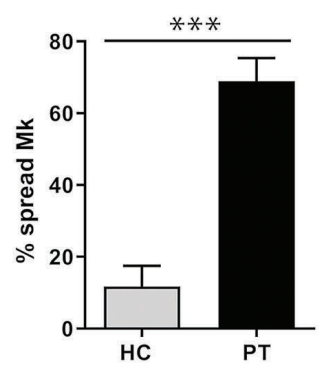

Figure 2. Analysis of proplatelet formation and spreading in adhesion to fibrinogen of megakaryocytes of the investigated patient. Megakaryocytes (Mk) were incubated on fibrinogen-coated coverslips for 16 hours at $37^{\circ} \mathrm{C}$ and $5 \% \mathrm{CO}_{2}$, fixed and stained with an anti- $\beta 1$-tubulin antibody (red). Hoechst (blue) was used for counterstaining nuclei. (A-D) Mk of the patient exhibited a markedly increased spreading, often with aberrant morphology, associated with defective extension of typical proplatelets. (A) (iii-vi) Representative examples of patient's Mk. (i-ii) Proplatelets-forming Mk of healthy controls processed in parallel are shown for comparison. Scale bars: $60 \mu \mathrm{m}$. (B) Proplatelet formation was quantified using fluorescence microscopy as the proportion of Mk displaying at least one proplatelet with respect to the total number of Mk. (C,D) Spreading was measured through image analysis as the average area covered by each Mk (C), and as the percentage of spread Mk with respect to the total number of Mk (D). The data reported with the histograms are expressed as means \pm standard deviation. $* * * P<0.001$, and $* P<0.05$ by two-tailed Student $t$-test. 
A
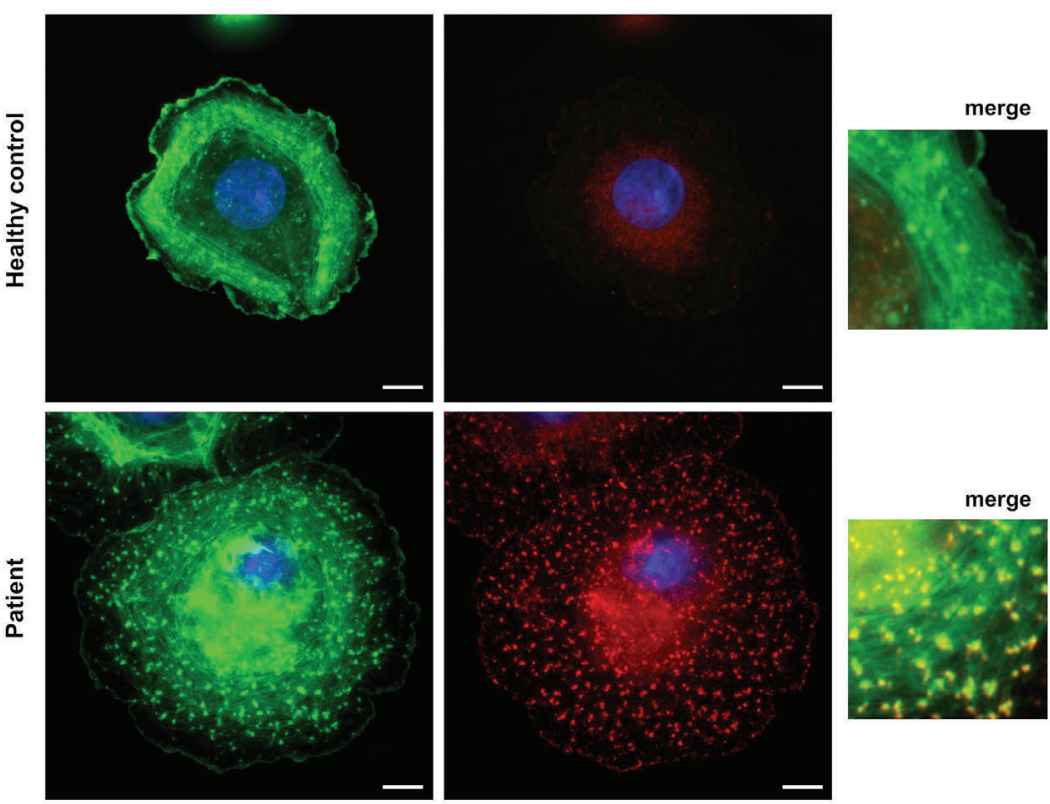

merge

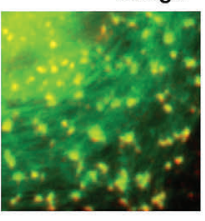

B

C
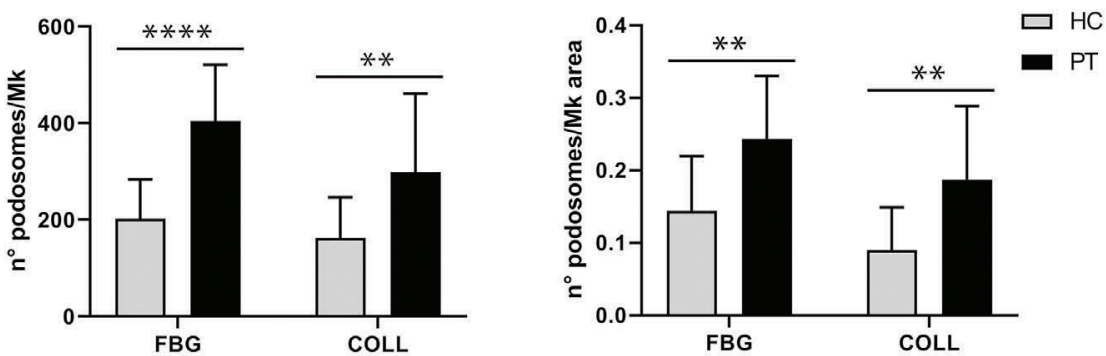

D

FIBRINOGEN

COLLAGEN

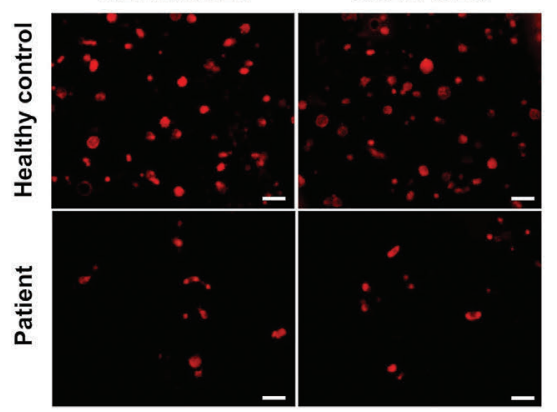

E

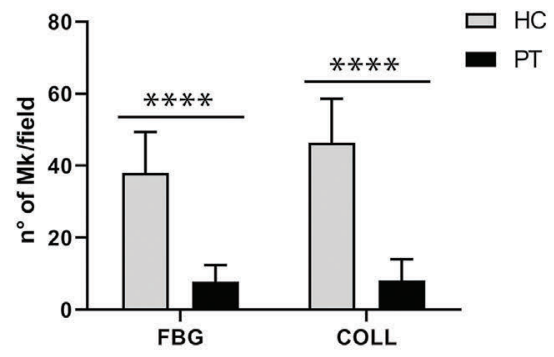

Figure 3. Analysis of podosome formation and of SDF1-driven migration of megakaryocytes of the investigated patient. (A-C) Megakaryocytes (Mk) were let adhere to fibrinogen or type I collagen, fixed, and double-stained for polymerized actin (phalloidin, green) and the active form of SRC (antibody selectively recognizing SRC phosphorylated at Tyrosine-419, red). Scale bars: $10 \mu \mathrm{m}$. (A) Patient's Mk exhibited a markedly increased number and density of podosomes that were recognized as actin-based focal adhesion structures (green); moreover, patient's Mk presented a strong positivity for the active form of SRC, which was highly and selectively concentrated in podosomes (red). The inserts on the right represent a merge of the images taken in the green and red signals, which demonstrates that the active form of SRC localizes in correspondence of podosomes recognized by phalloidin staining (yellow signal). (B,C) Podosomes formed on fibrinogen (FBG) and type I collagen (COLL) were quantified by image analysis of phalloidin-labelled Mk, as the number of podosomes per each Mk and the number of podosome per Mk area unit. (D-E) SDF1-driven migration of Mk was investigated using a modified transwell assay. Transwell systems having a polycarbonate membrane with an $8 \mu \mathrm{m}$ pore size were coated with fibrinogen or type I collagen. Aliquots of $4 \times 10^{3} \mathrm{Mk}$ were seeded in the upper chamber of the transwell insert, whereas the lower chamber was filled with medium containing $100 \mathrm{ng} / \mathrm{mL} \mathrm{SDF} 1$. After an incubation of 16 hour at $37^{\circ} \mathrm{C}$ and $5 \% \mathrm{CO}_{2}$, cells that had migrated to the lower face of the membrane were fixed and labeled with an anti- $\beta 1$-tubulin antibody (red) and counted using fluorescence microscopy. (D) Representative images of microscopic fields of migrated cells. Scale bars: $60 \mu \mathrm{m}$. (E) Mk migration was quantified as the number of migrated Mk per field by analyzing the entire polycarbonate membrane area. For each experiment, the assays were performed in triplicate wells for each condition. Data are expressed as means \pm standard deviation. $* * * * P<0.0001$ and $* * P<0.01$ by two-tailed Student $t$-test. 
therefore be rapidly progressive, underlining the importance of recognizing $S R C$-related thrombocytopenia among the other forms of inherited thrombocytopenia. In conclusion, the investigation of this patient provides novel information on the pathogenetic and clinical features of $S R C$-related thrombocytopenia and on the functions of SRC in human Mk.

Serena Barozzi, $1^{*}$ Christian A. Di Buduo, ${ }^{2,3^{*}}$

Caterina Marconi, ${ }^{4}$ Valeria Bozzi, ${ }^{1}$ Marco Seri, ${ }^{4}$

Francesca Romano, ${ }^{5}$ Alessandra Balduini, ${ }^{2,3}$ and Alessandro Pecci ${ }^{1}$

${ }^{1}$ Department of Internal Medicine, IRCCS Policlinico San Matteo Foundation and University of Pavia, Pavia; ${ }^{2}$ Department of Molecular Medicine, University of Pavia, Pavia; ${ }^{3}$ Laboratory of Biochemistry, Biotechnology and Advanced Diagnosis, IRCCS Policlinico San Matteo Foundation, Pavia; ${ }^{4}$ Department of Medical and Surgical Sciences, University of Bologna, Bologna and ${ }^{5}$ Postgraduate School of Pediatrics, University of Torino, Torino, Italy

${ }^{*} S B$ and $C A D B$ contributed equally as co-first authors.

Correspondence:

ALESSANDRO PECCI - alessandro.pecci@unipvit

doi:10.3324/haematol.2020.268516

Disclosures: no conflicts of interest to disclose.

Contributions: $S B$ and $C A D B$ performed research, analyzed and interpreted the laboratory data, wrote the manuscript; $C M, V B, M S$, performed research and analyzed and interpreted the laboratory data; $F R$ collected and interpreted the clinical data; $A B$ analyzed and interpreted the laboratory data and wrote the manuscript; AP designed research, analyzed and interpreted the data, and wrote the manuscript. All the authors critically revised the manuscript and approved the final version.

Acknowledgments: the authors would like to thank the patient and his family, and Dr. Emanuela Boveri, Dept. of Human Pathology, IRCCS Policlinico San Matteo Foundation, for helpful discussion on bone marrow histological findings.

Funding: This study has been supported by the Telethon Foundation (grant no. GGP17106, to AP); the IRCCS Policlinico San Matteo Foundation (to AP); the European Union (H2020-Project ID 767309, to $A B)$; the Italian Ministry of Health (GR-2018-12367235, to $C A D B)$.

\section{References}

1. Parsons SJ, Parsons JT. Src family kinases, key regulators of signal transduction. Oncogene. 2004;23(48):7906-7909.

2. Senis YA, Mazharian A, Mori J. Src family kinases: at the forefront of platelet activation. Blood. 2014;124(13):2013-2024.

3. Turro E, Greene D, Wijgaerts A, et al. A dominant gain-of-function mutation in universal tyrosine kinase SRC causes thrombocytopenia, myelofibrosis, bleeding, and bone pathologies. Sci Transl Med. 2016;8(328):328ra30.

4. De Kock L, Thys C, Downes K, et al. De novo variant in tyrosine kinase SRC causes thrombocytopenia: case report of a second family. Platelets. 2019;30(7):931-934.

5. Thiele J, Kvasnicka HM, Facchetti F, Franco V, van der Walt J, Orazi A. European consensus on grading bone marrow fibrosis and assessment of cellularity. Haematologica. 2005;90(8):1128-1132.

6. Obergfell A, Eto K, Mocsai A, et al. Coordinate interactions of Csk, Src, and Syk kinases with [alpha]IIb[beta] 3 initiate integrin signaling to the cytoskeleton. J Cell Biol. 2002;157(2):265-275

7. Mazharian A, Thomas SG, Dhanjal TS, Buckley CD, Watson SP Critical role of Src-Syk-PLC\{gamma\}2 signaling in megakaryocyte migration and thrombopoiesis. Blood. 2010;116(5):793-800.

8. Balduini A, Malara A, Pecci A, et al. Proplatelet formation in heterozygous Bernard-Soulier syndrome type Bolzano. J Thromb Haemost. 2009;7(3):478-484.

9. Bluteau D, Balduini A, Balayn N, et al. Thrombocytopenia-associated mutations in the ANKRD26 regulatory region induce MAPK hyperactivation. J Clin Invest. 2014;124(2):580-591.

10. Sabri S, Foudi A, Boukour S, et al. Deficiency in the Wiskott-Aldrich protein induces premature proplatelet formation and platelet production in the bone marrow compartment. Blood. 2006;108(1):134-140.

11. Schachtner H, Calaminus SD, Thomas SG, Machesky LM Podosomes in adhesion, migration, mechanosensing and matrix remodeling. Cytoskeleton (Hoboken). 2013;70(10):572-589.

12. Avecilla ST, Hattori K, Heissig B, et al. Chemokine-mediated interaction of hematopoietic progenitors with the bone marrow vascular niche is required for thrombopoiesis. Nat Med. 2004;10(1):64-71.

13. Marconi C, Di Buduo CA, LeVine K, et al. Loss-of-function mutations in PTPRJ cause a new form of inherited thrombocytopenia. Blood. 2019;133(12):1346-1357.

14. Ghalloussi D, Dhenge A, Bergmeier W. New insights into cytoskeletal remodeling during platelet production. J Thromb Haemost. 2019; 17(9):1430-1439.

15. Pecci A. Diagnosis and treatment of inherited thrombocytopenias. Clin Genet. 2016;89(2):141-153.

16. Williams $\mathrm{N}$, Levine RF. The origin, development and regulation of megakaryocytes. Br J Haematol. 1982;52(2):173-180. 